\title{
Automated, On-line, Calibration-Free, Particle Size Measurement using 3D Profile Data
}

\author{
M.J. Thurley \\ Luleå University of Technology, Luleå SE-97187, Sweden \\ email matthew.thurley@1tu.se \\ http://www.ltu.se/staff/m/mjt
}

\begin{abstract}
Optimisation and control of blasting, comminution and agglomeration processes is a complex task with large potential for gains in energy efficiency and productivity in the mining and aggregates industries. In order to realise these benefits, fully automated, non-contact, on-line particle size measurement technology is required to provide the necessary feedback. Results from two installations are presented for measurement of crushed rock on conveyor belt from a variety of sieved products between 0 and $100 \mathrm{~mm}$ and material from a primary crusher up to $250 \mathrm{~mm}$. Detailed sieve-size distribution results are presented with results calculated directly from the 3D surface profile data of the conveyor. No calibration against sieve samples has been performed clearly demonstrating the capability of the system to operate in a plug-and-play low maintenance setup. The technology measures 3D surface profile data which is used to delineate individual particles, identify non-overlapped particles, identify areas-of-fines, and produce a sieve-size distribution.
\end{abstract}

\section{INTRODUCTION}

\subsection{Overview}

In the mining and aggregate industries a great many processes affect, and are affected by particle size, including blasting, comminution, and agglomeration. As a result significant effort goes into measuring or estimating the size distribution of particulate material in order to evaluate and optimise production in terms of production rate and production costs (energy, material and equipment costs).

Mine and quarry operators want to measure the particle sizing results of all of these activities but sieving/screening is an imperfect assessment tool due to slow feedback, and inconsistent measurement due to operator fatigue, or variations in technique.

As a result there is an opportunity for on-line, non-contact, fully automated machine vision systems for measurement of particle size to facilitate evaluation and optimisation of mining and particle processes.

\subsection{Source of Error}

There are however, a number of sources of error relevant to techniques that measure only what is visible on the surface of a pile and it is necessary to consider these errors in order to ensure a measurement system can be stable, reliable, and trend in the right direction.

Particle delineation error refers to the inaccuracies of determining the correct delineation of all the individual particles in the measured surface (whether by an automatic computer program or manually). Significant error will render the measured surface largely meaningless as the particle delineation will bear little resemblance to the reality of what is on the surface of the pile. This error is significantly related to the type of surface measurement technique applied, whether photographic 2D, or 3D surface measurement. Given the impact of this error, evaluation of particle delineation is highly important. This error has been evaluated for the key particle delineation methods used here with 3D surface profile data by Thurley and $\mathrm{Ng}$ (2005).

Sub-resolution particle error, relates to the inability of an imaging system to see fine particles below the resolution of the sensor. For example, to detect an individual particle that is only represented by a few pixels or less in an image. These sub-resolution particles tend to be grouped into large regions and mis-sized as large rocks.

Segregation and grouping error, more generally known as the Brazil nut effect (Rosato et. al. 1987), describes the tendency of the pile to separate into groups of similarly sized particles. It is caused by vibration or motion (for example as rocks are transported by truck or conveyor) with large particles being moved to the surface. It is advisable to measure at a point early on the conveyor before the material has been subjected to excessive vibration and segregation.

Overlapped particle error describes the fact that many particles are overlapped (refer to 
Figure 1) and are only partially visible. A bias towards the smaller size classes results if overlapped are treated as small non-overlapped and sized using only their visible profile. This error can be overcome in piles of particulate material using classification algorithms based on 3D range data (Thurley and $\mathrm{Ng}$, 2008 ) successfully providing $82 \%$ classification accuracy on hold-out data (Andersson and Thurley, 2008).

Capturing error describes the varying probability based on size that a particle will appear on the surface of the pile. In simple terms, the larger a particle is, the more likely one is to be able to see some part of it on the surface. For example, if a single particle is as large as the height of the pile of material, then it will always be visible, whereas a very fine particle is almost certainly not visible. Thurley (2002) has explored capturing error in laboratory rock piles but it remains a source of error in this application.

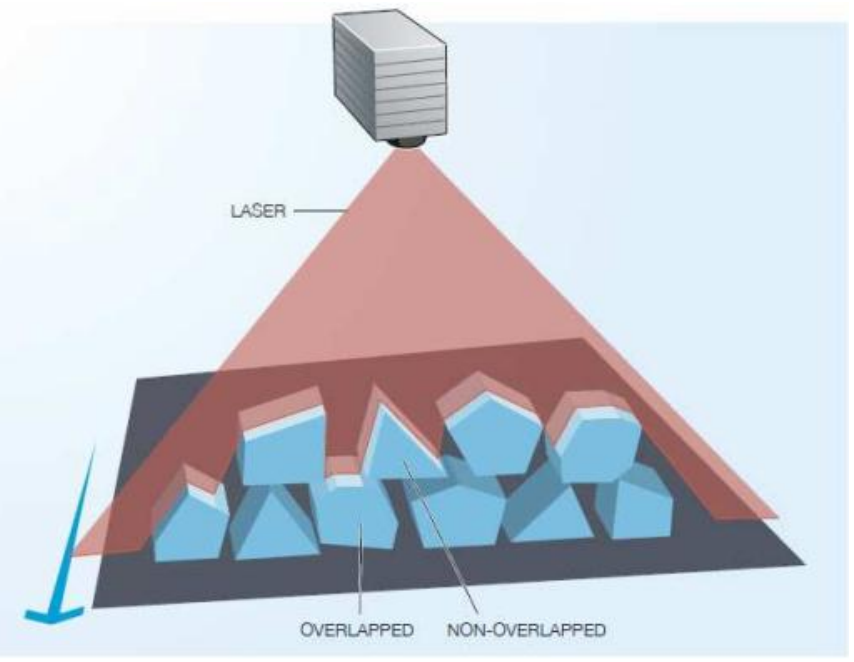

Figure 1. Illustration of overlapped and non-overlapped particles on conveyor passing through a sheet of laser light.

Profile error describes the fact that only one side (a profile) of a non-overlapped particle can be seen making it difficult to estimate the particles size. In the presented research the partial 3D surface profile of each non-overlapped particle and depth of the bed of material is used to estimate a 3D ellipsoidal volume of the particle.

Sample delimitation and extraction is an error relevant to all methods sampling from conveyor. Overcoming this error requires the correct delimitation of a belt section using two parallel transverse cuts across the belt, and correct extraction of particles where only particles whose centre-of-gravity is inside the delimited region are part of the sample. Refer to Pitard (1993) for a more thorough description.

Weight estimation error results from the fundamental difference between non-contact measurement and physical measurement. Size measurement using imaging identifies how many particles are observed, but manual sieving measures the weight of particles in each size class. Therefore it is necessary to have a method of mapping from numbers of particles to weight of particles in order to provide a measurement of size that industry understands and can use. The presented research uses volumetric estimation of each non-overlapped particle and assumes constant density within a sample to estimate a weight of each particle. Weight of fines is estimated based on the bulk volume of the observed areas-of-fines.

\subsection{Literature Review}

Particle size measurement using vision has been the subject of research and development since the 1980's (Carlsson \& Nyberg 1983, Ord 1988) with a legacy of predominantly photographic based systems with widely varying degrees of success and no general solution available on the market.

Photographic based 2D imaging systems are subject to significant particle delineation error due to uneven lighting conditions, excessive shadowing, and colour and texture variation in the material. Furthermore, photographic systems have no direct measure of scale, suffer from perspective distortion, lack the capability to distinguish between overlapped and non-overlapped particles, and do not demonstrate the ability to automatically detect visible fines in a realistic way. As a result photographic 2D systems typically require manual editing of the particle delineation to provide a reasonable estimation.

In 1996 the Fragblast 5 conference held a workshop on the measurement of blast fragmentation with the stated aim "to review the state-of-the-art in image processing as applied to measurements of rock fragmentation (Franklin \& Katsabanis 1996). Prior to Fragblast 10, this was the most thorough review of systems (photographic 2D) for this purpose. The keynote address from this workshop by Cunningham (1996) is particularly critical in its review of image analysis systems for rock fragmentation and in highlighting common conclusions. Cunningham (1996) noted "that almost every such paper includes a statement such as the system is practical for suitable use ... although further work is required". Furthermore, "graphs of system output versus sieving results are described as providing good or reasonable correlation" but that "it is often difficult to concur with the sentiments expressed".

In their review of the Split commercial photographic based 2D system Potts \& Ouchterlony (2005) report that for their application the system erroneously assumes the resultant size distribution is uni-modal and they conclude by expressing strong reservations saying 2D "imaging has a certain but limited usefulness when measuring the fragment size distribution in a muckpile or from a belt in an accurate way. It could probably detect rough tendencies in fragmentation variations, if the lighting conditions 
do not vary too much, and if cover glasses for camera lenses are kept clean".

Comparisons have been published between photographic methods and sieving, specifically Wang \& Stephansson (1996) and Fernlund [1998]. Wang \& Stephansson (1996) performed image analysis on overlapping fragments and reported "a systematic error compared to sieving analysis'. This error is in part due to the inability of photographic 2D image analysis and particle delineation to provide the information necessary to distinguish between entirely (non-overlapped) and partially visible (overlapped) rocks.

There are a number of publications relating to $3 \mathrm{D}$ size measurement including Noy (crushed rock, 2006), Frydendal \& Jones (sugar beets, 1998), Kim et. al. (river rock, 2003), Lee et. al. (2005), and Thurley $(2002,2009,2011)$. However, Frydendal and Jones (1998) and the presenting author (Thurley \& $\mathrm{Ng}, 2008)$ are the only publications to remove the bias resulting from overlapped particles. For conveyor belt applications some publications recommend installing a mechanical vibration feeder (Kim et. al. 2003, Lee et. al. 2005) to separate rocks and prevent particle overlap. If there exists the possibility to install a vibration feeder at full-scale in the process it would simplify image analysis, and would likely improve results but with the added cost and maintenance of the additional equipment.

The focus of this research is on systems for online measurement of the particle bed that are noncontact, that is they do not require any additional material handling. If non-contact measurement is required then one must consider overlapped particle error and account for overlapped and nonoverlapped particles. Furthermore, in some circumstances, such as examination of rocks in inproduction excavators (Thurley 2009), there is no other option than to account for overlapped and nonoverlapped particles.

\subsection{Measurement System Overview}

This research uses an industrial measurement system on conveyor belt based on laser triangulation (a projected laser line and camera at an offset angle) collecting highly accurate 3D profiles of the laser line approximately $3000 \mathrm{~Hz}$. This high speed ensures a high density of 3D point data with a spatial resolution between consecutive points in the direction of the belt of approximately $1 \mathrm{~mm}$ for a conveyor belt is running at $3 \mathrm{~m} / \mathrm{s}$. One measurement system is installed at a limestone quarry on the conveyor belt used for ship loading and measures the material on the belt during loading every 30 seconds. This allows measurement of a variety of screened and stockpiled products with a narrow range of sizes and it is against these products the measurement system performance is evaluated. Figure 2 shows an image of the installed laser and camera mounted above the conveyor belt as illustrated in Figure 1.

Using the same laser triangulation technology it is also possible to collect measurement points at $0.1 \mathrm{~mm}$ spatial resolution and $0.005 \mathrm{~mm}$ depth resolution as we have done on steel slabs. Using such a setup it should be possible to confidently measure particles with a lower size limit of $1 \mathrm{~mm}$ and be able to detect overlapped and non-overlapped particles on conveyor belt. An equivalent reduction in measurement speed (one tenth conveyor belt speed) is necessary such as $0.3 \mathrm{~m} / \mathrm{s}$ but it is theoretically possible to improve this using higher power lasers (beyond class 3B).

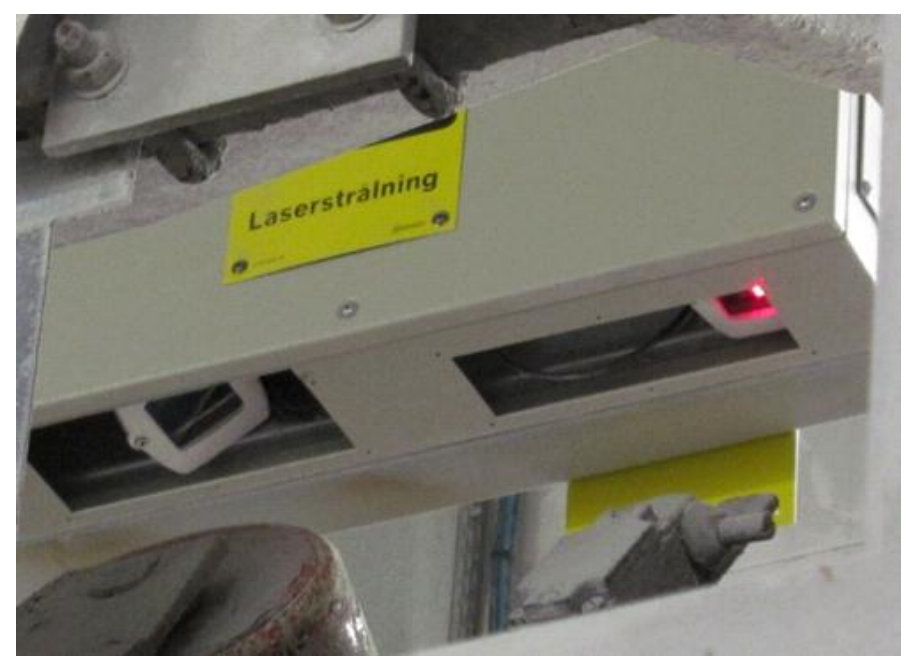

Figure 2. Measurement system showing laser source (right side) projecting a laser plane and digital camera (left side) observing the interaction of the laser and the particles on the conveyor.

The presented analysis algorithms are not particularly dependent on the laser triangulation measurement technology. Any other technique for capturing 3D surface data of a particle pile, such as stereo photogrammetry, laser scanners, or time-of-flight 3D cameras could also be used.

The computational speed of the analysis process is approximately 20 seconds on a $2.7 \mathrm{GHz}$ Intel i7 CPU processing a data set of approximately 1 million 3D points $(1.5 \mathrm{~m}$ long, $800 \mathrm{~mm}$ wide section of the belt). Furthermore, advances in both algorithmic efficiency and hardware are both available to improve computational time as the image processing operations are readily parallelisable. Computation time could be reduced down to the order of few seconds or less for rapid automatic control applications. Once the analysis can be performed in a under a second then it will to become possible to measure and analyse the entire surface of the material passing under the measurement system.

It is necessary to consider the largest particle size that should be measured and how many of these particles need to be measured to get a statistically valid measurement size. Given the circumstances in this 
application we currently use a $1.5 \mathrm{~m}$ long, $800 \mathrm{~mm}$ wide conveyor belt sample is sufficient given the material top size of approximately 90 to $100 \mathrm{~mm}$.

\subsection{Research Background}

The presented research builds upon a series of achievements and research developed on both laboratory rock piles and industrial application.

An industrial measurement system on conveyor belt for iron ore pellets (Thurley \& Andersson 2007) has been developed using the same laser triangulation measurement technology. The high speed camera system ensures we have a high density of 3D point data at a spacing between consecutive points in the direction of the belt of approximately $0.5 \mathrm{~mm}$. This high data density has at least two advantages. Firstly it allows us to detect small sliver regions or crescent-like regions of overlapped particles and ensure that they are not merged into other regions. Secondly, it ensures high resolution when it comes to measuring the size of each iron ore pellet allowing a size distribution with very fine spacing of $5,8,9$, $10,11,12.5,13,14$, and $16+\mathrm{mm}$ size classes.

One of the key criteria for particle size measurement is therefore high data density as it defines the capacity to detect small overlapped particles, the lower limit on individual particles that can be reliably detected, and the resolution of size classes detectable.

In addition a demonstration project for size measurement of rocks in underground LHD excavator buckets (Thurley 2009) has been performed. A 3D vision system based on laser scanners was installed on the tunnel roof in a production area of an underground iron ore mine with $3 \mathrm{D}$ surface data of the bucket contents being collected as the LHD unit passes beneath. The project successfully demonstrated fragmentation measurement of the rocks in the bucket, identifying overlapped rocks, nonoverlapped rocks, and estimation of the sieve-size of the visible rocks. The potential to identify areas-offines and prevent misclassification of such regions as large rocks was shown, and used in the presented research.

This research presents advances in three areas;

1. development of an image analysis strategy that works across a broad range of material scales from fine particles $(0.2 \mathrm{~mm})$, up to (but not limited to) $250 \mathrm{~mm}$. A summary of these results is presented but the details of this process are outside the scope of this paper. Background research has been previously published by Thurley (2011) and Thurley \& Ng (2005).

2. validation of the capacity to detect areas-of-fines using a variety of test data, and

3. development of a volumetric based particle sizing method that individually sizes each non- overlapped particle, detects areas-of-fines, and incorporates this information into a sieve-sizedistribution (by volume). Assuming constant density of the material, this provides an estimation of the sieve-size-distribution by weight.

\section{METHOD \& RESULTS}

\subsection{Data Collection}

In the installation at the limestone quarry the majority of particles are above $10 \mathrm{~mm}$ and the system was originally installed to only measure particles at $10 \mathrm{~mm}$ or larger. Therefore the system collected 3D data points at a spatial resolution of $1 \mathrm{~mm}$ to ensure sufficient data resolution to identify these particles. This spatial resolution is maintained for the second measurement system located after the crusher so we can easily compare results between the two systems.

A number of different products (size ranges of material) were measured in order to evaluate the system at various scales. Particularly important was to test the system with material below the measurement limit of the system. As the system collects 3D data points with an $(\mathrm{x}, \mathrm{y})$ spatial resolution of $1 \mathrm{~mm}$ it is not possible for the system to identify individual particles close to this spatial resolution. As it is necessary to be able to identify individual particles, and small parts of overlapped particles, it is reasonable to expect that the system cannot detect individual particles below $5 \mathrm{~mm}$. Therefore a data set comprising particles in the range $0-2 \mathrm{~mm}$ was obtained to ensure that there should not be any particles large enough for the system to identify. Measurements were performed on stockpiled products in the size range $0-2,10-25,20-40,40-70,60-90 \mathrm{~mm}$, and then on three data sets from a primary crusher with material in the range $0-250 \mathrm{~mm}$.

\subsection{Particle Delineation}

The first step is to perform the detailed particle delineation (image segmentation) to identify the individual rock particles. These techniques have been applied to laboratory rock piles (Thurley \& $\mathrm{Ng}$ 2005), in an industrial pellet measurement system (Thurley \& Andersson 2007) and rocks on conveyor (Thurley 2011), the latter of which forms the basis of the presented results. The technique is predominantly based on morphological image processing (Dougherty \& Lotufo 2003, or any broad textbook on image processing), based largely on various edge detection techniques to facilitate seed formation for the watershed segmentation algorithm (Beucher \& Meyer 1992, Dougherty \& Lotufo 2003). 

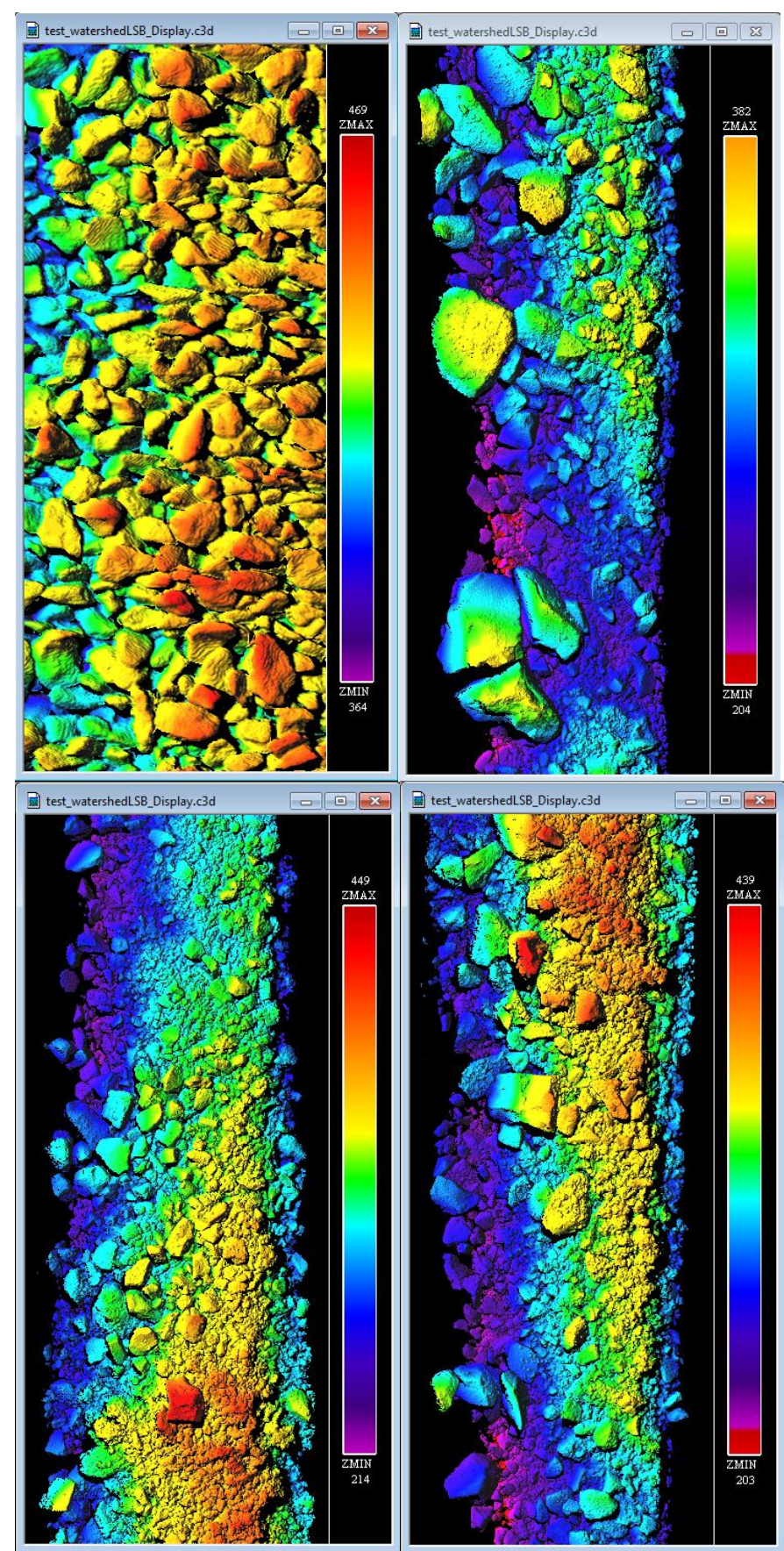

Figure 3. 3D surface profile of rocks on conveyor. 20-40mm product (top left), and three data sets from the output of a primary crusher in the range $0-250 \mathrm{~mm}$.

Figure 3 shows images of the 3D surface profile of rocks on the conveyor for the $20-40 \mathrm{~mm}$ data set, and 3 sets from the $0-250 \mathrm{~mm}$ crusher feed.

Figure 4 shows the automated particle delineation results. Particles have been removed from the delineation on each end of the conveyor in accordance with the sampling and delineation strategy suggested by Pitard (1993), removing all particles whose centre-of-mass is not placed within a defined transverse delineation zone.

The particle delineation is processed further to identify areas-of-fines (Thurley 2009), and nonoverlapped particles (Thurley \& Ng 2008). These methods work by following the perimeter of every
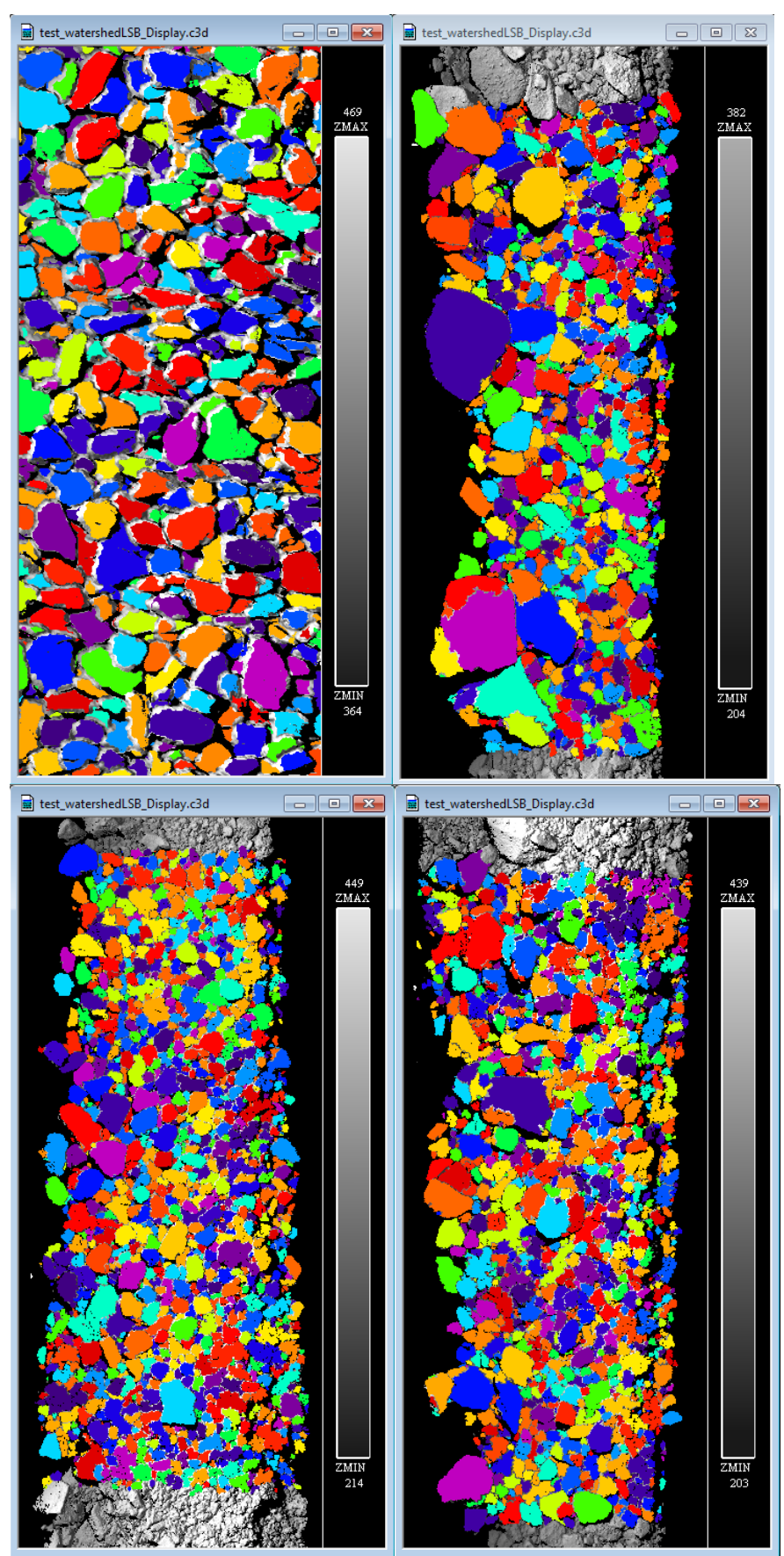

Figure 4. Fully automated particle delineation. $20-40 \mathrm{~mm}$ product (top left), and three data sets from the output of a primary crusher in the range $0-250 \mathrm{~mm}$.

delineated particle and examining the depth variation in the 3D surface data. Regions that are nonoverlapped will be consistently above their neighbour regions. Regions that are rocks will typically have large depth variations around the perimeter, whereas regions that are fines will typically have small depth variations. This is because the areas-offines are typically over-segmented (contain many seemingly random regions) and the areas-of-fines have slowly varying surface topology (changes in depth). 


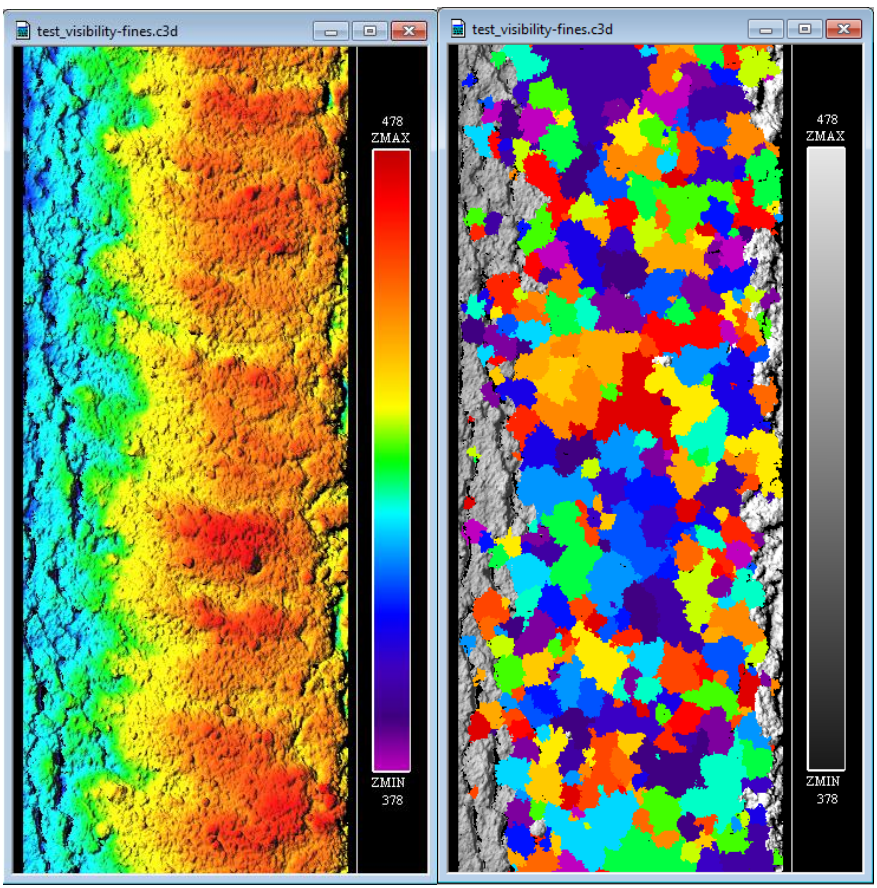

Figure 5. 3D surface profile data and automated delineation for $0-2 \mathrm{~mm}$ particles, showing large regions detected as areas-offines.

Figure 5 illustrates this capacity to overcome $s u b$ resolution particle error and automatically detect areas-of-fines, with the $0-2 \mathrm{~mm}$ data set shown on the left, with the regions from the delineation that were classified as areas-of-fines shown on the right in shaded/coloured blobs. This ensures that areas-offines are not mis-sized as large particles. There are regions on the left and right edges of the right data set that were not classified as fines. The data in these areas appears to have some vertically oriented ridges in the data that look significantly like rock structures and so are not detected as fines. An additional data set where the belt was more heavily loaded with the $0-2 \mathrm{~mm}$ product did not have these ridges and when analysed $98 \%$ of cumulative sieve-size distribution was classified as below $5 \mathrm{~mm}$. Unfortunately this data was lost on a stolen computer and at the time of writing additional $0-2 \mathrm{~mm}$ data sets have not been collected.

Figure 6 shows all particles automatically identified as non-overlapped and illustrates the capacity to overcome overlapped particle error preventing missizing of overlapped particles as small size classes.

Being able to largely eliminate these two sources of error removes error that biases the results towards smaller sizes (overlapped particle error) and error that biases towards large sizes (sub-resolution particle error). A system with opposing sources of error will tend to be unstable and lack the capacity to trend in the right direction. As we can eliminate these sources of error, the presented research has been consistently demonstrated to trend in the right direction.

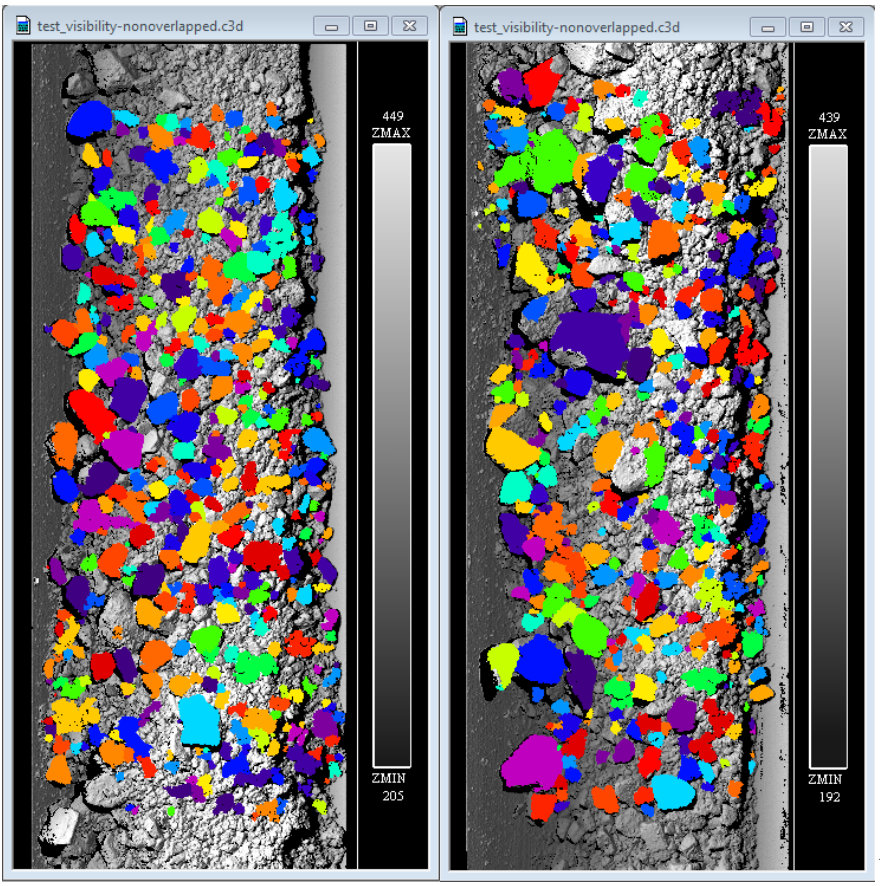

Figure 6. Automatically identified non-overlapped particles. Left image corresponds to Figure 3 bottom left. Right image corresponds to Figure 3 bottom right.

\subsection{Particle Classification \& Sizing}

Particle sizing represents a significant improvement in this research to previous work. A new volumetric based sizing has been applied that uses the 3D profile data of each particle, and as a result no "calibration" or statistical matching to expected sieve results was performed in this research.

Particle sizing is performed in the following way;

1. Each region in the particle delineation is classified as fines, overlapped, or non-overlapped based on the methods published earlier by Thurley $\& \mathrm{Ng}$ (2008) and Thurley (2009).

2. Each region identified as fines is converted into a volume based on the 3D surface profile and the depth of the material on the belt at each point in the 3D surface profile (from a measurement of the weighted-down empty conveyor). The volume beneath the region is assumed to be fines. The fines volume is allocated to a particle size of less than $5 \mathrm{~mm}$ as this is the predefined minimum size in these cases.

3. Each region identified as overlapped is currently ignored. These regions are analogous to ice-bergs and are a more difficult prospect for sizing. Identifying and ignoring these particles prevents missizing them are smaller particles.

4. Each region identified as non-overlapped is processed as follows;

a) The best-fit-rectangle in the horizontal plane is calculated for that region. From the length and 
width of this rectangle a major axis and a minor axis for the particle is defined, denoted major, minor.

b) The 3D surface profile of the particle is analysed to calculate the maximum height, denoted $Z_{\max }$ and minimum height of the particle, denoted $Z_{\min }$

c) The height of the particle $H$ is estimated to be double the known height of the particle, unless this height would make the particle extend below the conveyor belt. The height is approximated by equation (1) as the minimum of the following two expressions where $D_{z \min }$ is the depth of the material on the conveyor at the location of $Z_{\min }$

$H=\min \left[2 *\left(Z_{\max }-Z_{\min }\right\}, D_{z \min }+Z_{\max }-Z_{\min }\right]$

d) The volume of the particle is approximated using an ellipsoid with axes $H$, major, minor. Therefore the volume $\mathrm{V}=(4 / 3) \pi . H . m a j o r . m i n o r$

e) The sieve-size of the particle is approximated as the minimum value of $H$ and minor. Note that it would be more appropriate to estimate the sievesize as the side length of the smallest square that can contain an ellipse with $H$, minor as its two principle axes and we will use this in the future.

f) All particles with a sieve-size less than $5 \mathrm{~mm}$ are added to the fines volume.

5. The sieve-size-distribution is calculated by ordering all of the particles by their sieve-size (starting with the fines volume) and calculating the cumulative sum of all of their volumes. Figure 7 and Figure 8 graph these sieve-size-distributions, illustrating the lack of predefined sieve-size-classes in the graphs. This allows any arbitrary sievesize-classes to be determined after the analysis. Table 1 shows the 20\%, 50\% and 80\% passing values for the curves in both figures.

Performing the analysis on over 6 hours of ship loading data in which the $40-70$ and $60-90 \mathrm{~mm}$ products were being loaded produces the graph shown in Figure 9 . Only the 20,50 , and $80 \%$ passing values are plotted and one can clearly see when the loading changes and identify the product being loaded at any given measurement based on the $20 \%$ and $80 \%$ passing values.
Size Distribution Crusher Output

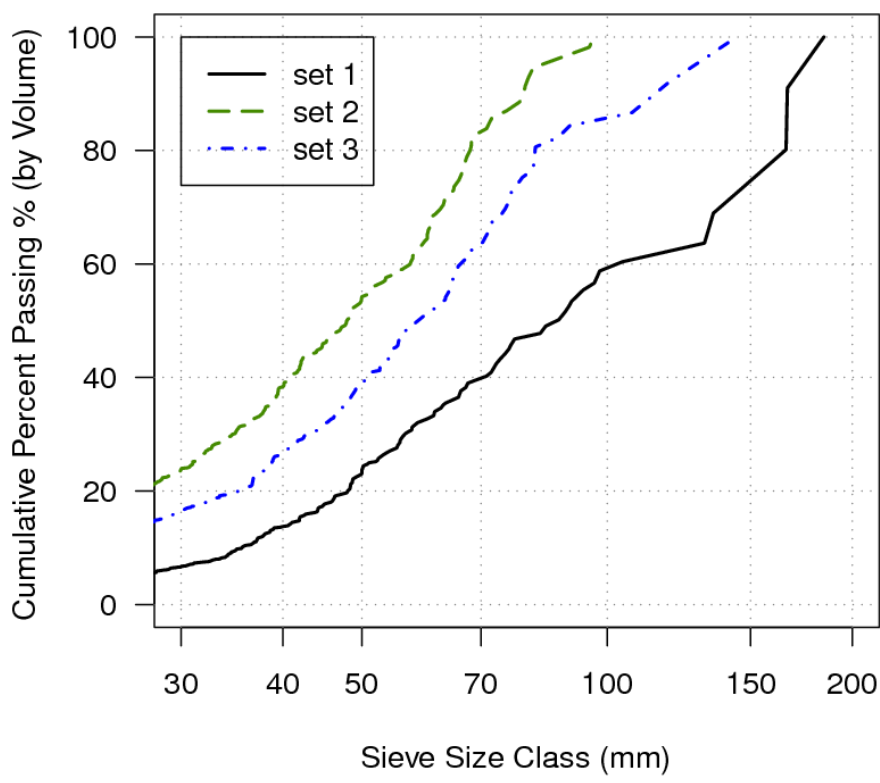

Figure 7. Calculated cumulative sieve-size distribution for the three crusher data sets in Figure 3. Set 1 (solid line) is the top right image in Figure 3, set 2 (dashed line) is the bottom right image, and set 3 (dot-dash line) is the bottom left image.

\section{Size Distributions - Sieved Products}

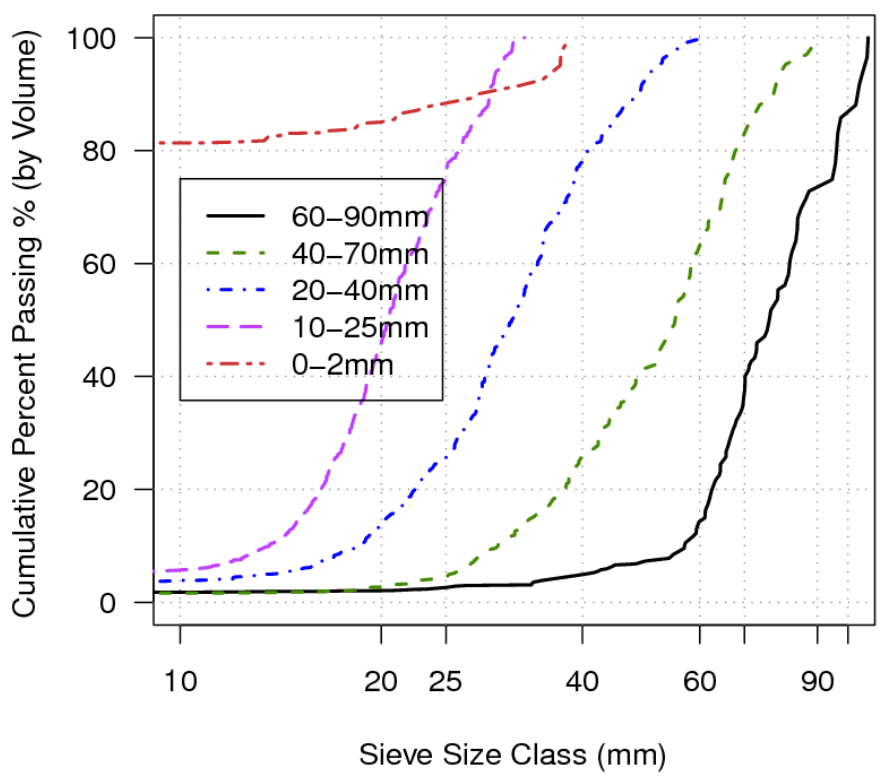

Figure 8. Calculated cumulative sieve-size distribution for the stockpiled products. From the left most distribution curve the product size is $0-2,10-25,20-40,40-70$, and $60-90 \mathrm{~mm}$ on the far right.

Table 1. Calculated 20, 50, 80 percent passing values (mm) for each product.

\begin{tabular}{lcccccccc}
\hline Sieve-size (mm) & $\mathbf{0 - 2}$ & $\mathbf{1 0 - 2 5}$ & $\mathbf{2 0 - 4 0}$ & $\mathbf{4 0 - 7 0}$ & $\mathbf{6 0 - 9 0}$ & & $\mathbf{0 - 2 5 0}$ & \\
\hline $\mathbf{8 0 \%}$ passing & 5 & 26.2 & 40.7 & 67.8 & 96.1 & 165 & 67.8 & 81.5 \\
$\mathbf{5 0 \%}$ passing & 5 & 20.5 & 31.4 & 55.0 & 76.0 & 84.1 & 47.9 & 58.1 \\
$\mathbf{2 0 \%}$ passing & 5 & 16.2 & 22.5 & 37.4 & 62.5 & 47.9 & 27.0 & 35.1 \\
\hline
\end{tabular}




\section{Cumulative Size Distribution}

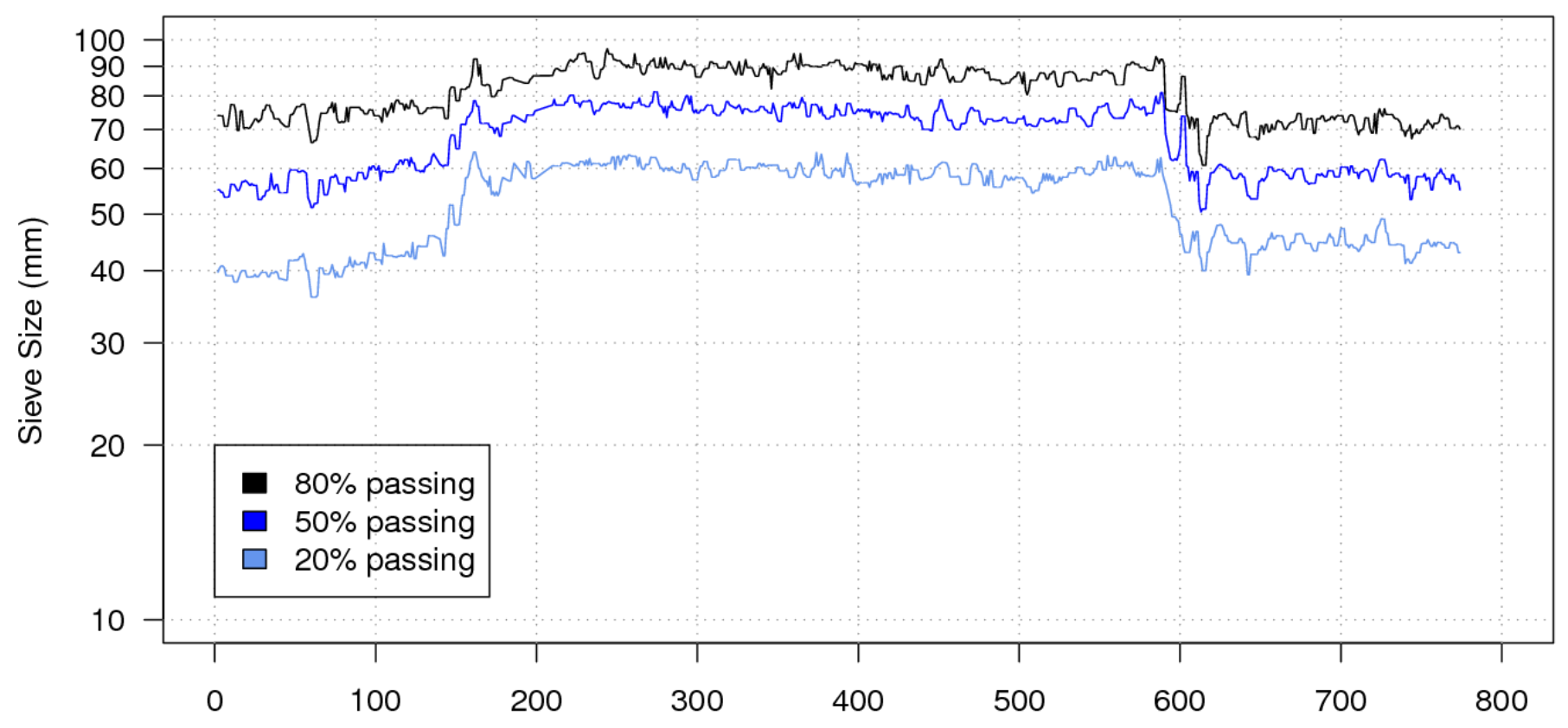

Measurement Number During the Day

Figure 9. Over 6 hours of measurement data (767 measurements) during loading of the 40-70mm and 60-90mm products.

\section{DISCUSSION}

\subsection{System Results}

The presented research demonstrates a non-contact, automated particle delineation and analysis technique that uses 3D surface profile data on a broad range of material sizes and applies a new sizing strategy to directly calculate the sieve-sizedistribution by volume (approximating weight). This allows the capability to install the system without any need to calibrate against manual sieve samples.

The results show a strong relationship to the listed product size range for the pre-sieved products and it is clear from observation of the 3D data in Figure 3 and the cumulative size distributions in Figure and Figure 8 that the results trend in the right direction. That is, when large material is on the belt, the size distribution result is larger.

Comparison against manual sieving results is not yet available but will be performed in future work to fine-tune the size estimation methods and investigate the system accuracy.

Based on observation of the results in Table 1, Figure 8 and Figure 9 it is clearly identifiable which sieve curve corresponds to which product, and we can observe that the $20 \%$ and $80 \%$ passing values come reasonably close to the listed product intervals.

As a result, the presented system could readily be used for measurement, feedback, and even control of crushing, grinding, and agglomeration processes. If located before or after a primary crusher the presented system could also provide feedback to blasting.

The capacity to automatically distinguish between overlapped, non-overlapped and areas-of-fines, and to treat each of these three cases differently is a unique contribution of this body or work and it allows a number of sources of error to be addressed.

\subsection{Sources of Error}

The following summary identifies how these sources of error have been mitigated, addressed, or ignored;

Particle delineation error is not the subject of this paper, but a detailed investigation is performed by Thurley \& Ng (2005). One can note however, that using 3D surface profile data eliminates particle delineation problems due to shadows, and colour variation in the material which can affect photographic 2D systems.

Sub-resolution particle error is mitigated by this implementation as areas-of-fines can be automatically detected and sized as fines instead of being missized as large particles.

Segregation and grouping error remains a constant source of error but appears not to be particularly significant for the stockpiled products. It may have a significant but as yet unmeasured impact on the case of the crusher material but as noted above, these results clearly trend in the right direction allowing the possibility of process control. 
Overlapped particle error is entirely mitigated by the presented system as it prevents mis-sizing of overlapped particles as smaller particles.

Capturing error is ignored but could be further investigated in future work if increased accuracy is necessary.

Profile error remains a relevant source of error, as it is not clear how accurate our 3D volumetric estimation is for each individual particle. However, when the sample is considered as a whole, the sizedistribution results suggest the profile error is not a significant problem. Further investigation could be performed in the future to evaluate and improve accuracy.

Sample delimitation and extraction is not a significant source of error as a correct delineation and extraction based on centre-of-mass is performed.

Weight estimation is mitigated in this implementation as volumetric estimation of each particle is performed. The assumption of constant density within a sample seems reasonable but will lead to a source of error. Weight estimation in this case reverts back to profile error and the correct estimation of the particles actual 3D volume.

Surface analysis is by definition a source of error. There is no avoiding this error, but there is the possibility to mitigate it further. Future work will include collecting the particle size information and bulk weight information from belt scales. With this information it may be possible to estimate the proportion of hidden fines in the material given the observed particle size distribution and any discrepancy between expected weight and measured weight.

\section{CONCLUSION}

This research demonstrates algorithms and a measurement system for automated, non-contact, on-line, particle size measurement that can be used without the need to calibrate or statistically correct the measurement results against a priori sieving results. Moreover the research demonstrates how relevant sources of error are addressed and mitigated in order to ensure a system that can be reliable and trend in the right direction. Without mitigating these errors, particularly overlapped particle error, and subresolution particle error, it seems unrealistic to expect reliable results. Key outcomes include;

1. The core capabilities to;

a. automatically delineate particles,

b. classify delineated regions as overlapped particles, non-overlapped particles, or areas-of-fines, and

c. size particles based on volume in order to estimate the sieve-size-distribution

2. Sieve-size-distribution results that are close to expected product sizes,

3. Results that trend in the right direction,
4. Results that are clearly appropriate for feedback and control of industrial processes, and

5. A system that can work without any calibration against manual sieving making it easy to setup and maintain.

The presented algorithms are readily applicable to other mining processes, such as the feed hopper to a primary crusher, or in excavator buckets, shovels, drawpoints, and muckpiles, using an alternate appropriate method of collecting 3D surface profile data in that environment.

Some further development and testing is planned including;

1. Large scale testing collecting weeks of production data and comparison to daily manual sieving results.

2. Estimation of hidden fines may be possible based on the presented technique coupled with on-line weight information from belt scales.

3. Improvements in computational speed so that measurements can be performed in a few seconds or faster in order to allow fast automatic control strategies.

4. Improvements and tuning of the sieve-size calculation.

\section{ACKNOWLEDGEMENTS}

The author would like to thank all those who have supported this research over the last 15 years. It is the beginning of the end of the image analysis research effort for this method, and sincere thanks go to all those who have been involved, specifically; Luleå University, ProcessIT Innovations, Nordkalk, MBV-Systems AB, MinBaS, LKAB, SSAB, New Boliden, VINNOVA, EU INTERREG IVA Nord, Softcenter AB, CSIRO Exploration \& Mining, Monash University, Sven Molin, Olov Marklund, Pär-Erik Martinsson, Anders OE Johansson, Håkan Pihl, Marianne Thomaeus, John-Erik Larsson, Jan Nyström, Peter Wedin, Tobias Andersson, Johan Carlson, Kim Ng, John Minack, Cathy Wilkinson, Bruce Hobbs, Alison Ord, Lawrence Cheung, David LeBlanc, Paul Maconochie, Anthony Maeder, and family, and to those that have been missed, sincere apologies. 


\section{REFERENCES}

Andersson T. \& Thurley M.J. 2008, Visibility classification of rocks piles, in Proceedings of the 2008 Conference of the Australian Pattern Recognition Society on Digital Image Computing Techniques and Applications (DICTA 2008). Australian Pattern Recognition Society, pp. 207-213

Beucher S. \& Meyer F. 1992, Mathematical Morphology in Image Processing, Marcel Dekker, New York, Chapter 12, pp. 433-481.

Carlsson O. \& Nyberg L. 1983, A method for estimation of fragmentation size distribution with automatic image processing," in Proceedings of the First International Symposium on Rock Fragmentation by Blasting - FRAGBLAST, Luleå, Sweden, August 1983, pp. 333-345

Cunningham C.V.B. 1996, Keynote address: Optical fragmentation assessment - a technical challenge, in Measurement of Blast Fragmentation-Proceedings of the FRAGBLAST 5 Workshop, Balkema, pp. 13-19.

Dougherty E.R.\& Lotufo R.A. 2003, Hands-On Morphological Image Processing. SPIE - The International Society for Optical Engineering, vol. TT59

Fernlund J.M.R 1998, The effect of particle form on sieve analysis: a test by image analysis, Engineering Geology, 50 pp. 111-124.

Franklin J.A. \& Katsabanis T. 1996, (eds.), Measurement of Blast Fragmentation-Proceedings of the FRAGBLAST 5 Workshop, Balkema

Frydendal I. \& Jones R. 1998, Segmentation of sugar beets using image and graph processing, in ICPR 98 Proceedings 14th International Conference on Pattern Recognition, vol. II, Brisbane, Australia, August 1998, pp. 16-20.

Kim H., Haas C., Rauch A., \& Browne C. 2003, 3D image segmentation of aggregates from laser profiling, Computer Aided Civil and Infrastructure Engineering, pp. 254-263

Lee J., Smith M., Smith L., \& Midha P. 2005, A mathematical morphology approach to image based 3D particle shape analysis, in Machine Vision and Applications, vol. 16(5). Springer-Verlag, 2005, pp. 282-288

Ord, A. 1988, Real-time image analysis of size and shape distributions of rock fragments. Explosives in Mining Workshop, Melbourne, Victoria, November 1988. The AusIMM 115-119

Noy M.J. 2006, The latest in on-line fragmentation measurement - stereo imaging over a conveyor, in Proceedings of the Eighth International Symposium on Rock Fragmentation by Blasting - FRAGBLAST 8, May 2006, pp. 61-66.

Pitard E. 1993, Pierre Gy's sampling theory and sampling practice: heterogeneity, sampling correctness and statistical process control. CRC Press, 1993

Potts G. \& Ouchterlony F. 2005, The capacity of image analysis to measure fragmentation, an evaluation using split desktop. Swebrec - Swedish Rock Breaking Institute, Technical Report, 2005, ISSN 1653-5006.

Rosato A., Strandburg K., Prinz F., \& Swendsen R., 1987 Why the brazil nuts are on top: Size segregation of particulate matter by shaking, Physical Review Letters, vol. 58, no. 10, pp. $1038-1040$

Thurley M.J. 2002, Three dimensional data analysis for the separation and sizing of rock piles in mining, Ph.D. dissertation, Monash University, December 2002

Thurley M.J. 2009, Fragmentation size measurement using 3D surface imaging (in lhd buckets)," in Proceedings of the Ninth International Symposium on Rock Fragmentation by Blasting - FRAGBLAST 9, Granada, Spain, September 2009, pp. 133-140

Thurley M.J. 2011, Automated online measurement of limestone particle size distibutions using $3 \mathrm{~d}$ range data, Journal of Process Control, vol. 21, no. 2, pp. 254-262
Thurley M.J. \& Andersson T. 2007, An industrial 3d vision system for size measurement of iron ore green pellets using morphological image segmentation, Minerals Engineering, vol. 21 , no. 5 , pp. $405-415$

Thurley M.J. \& Ng K. 2005, Identifying, visualizing, and comparing regions in irregularly spaced $3 \mathrm{~d}$ surface data, Computer Vision and Image Understanding, vol. 98, no. 2, pp. 239-270

Thurley M.J. \& Ng K. 2008, Identification and sizing of the entirely visible rocks from segmented $3 \mathrm{~d}$ surface data of laboratory rock piles, Computer Vision and Image Understanding, vol. 111 , no. 2 , pp. 170-178

Wang W.X., Stephansson O. 1996, Comparison between sieving and image analysis of aggregates, in Measurement of Blast Fragmentation-Proceedings of the FRAGBLAST 5 Workshop, Balkema,pp. 141-148.

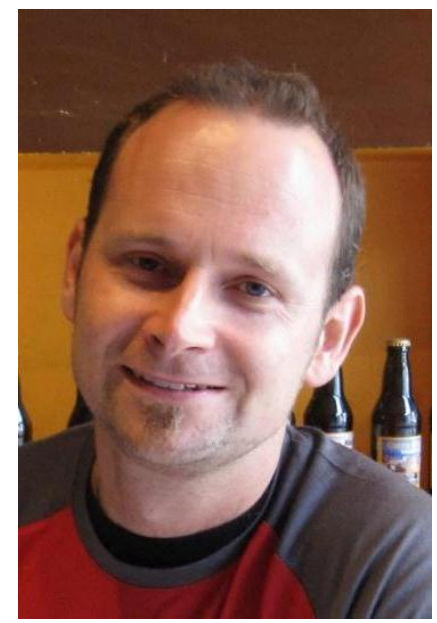

Matthew J. Thurley is associate professor and founder of an emerging industrial image analysis group at $\mathrm{Lu}-$ leå University of Technology. He has a strong focus on image analysis and algorithms for fully automated online particle size measurement. The group focuses on industrial measurement projects mainly within the mining and steel industries. 